Research Article

\title{
High Expression of JMJD4 Is a Potential Diagnostic and Prognostic Marker of Renal Cell Carcinoma
}

\author{
Hao Yan $\mathbb{D}^{1},{ }^{1}$ Yewei Bao, ${ }^{2}$ and Zongming Lin $\mathbb{D}^{1}$ \\ ${ }^{1}$ Department of Urology, Zhongshan Hospital, Fudan University, China \\ ${ }^{2}$ Department of Urology, Changhai Hospital, Naval Medical University, China \\ Correspondence should be addressed to Zongming Lin; lin.zongming@zs-hospital.sh.cn
}

Received 6 October 2021; Accepted 18 November 2021; Published 24 December 2021

Academic Editor: Zhongqiu Xie

Copyright (c) 2021 Hao Yan et al. This is an open access article distributed under the Creative Commons Attribution License, which permits unrestricted use, distribution, and reproduction in any medium, provided the original work is properly cited.

\begin{abstract}
Histone demethylase JMJD4 is a burgeoning tumor marker, which has been proven to be associated with colon cancer, but the role it plays in kidney cancer has not yet been investigated. In the present study, we evaluated whether JMJD4 can be a prognostic marker of patients with clear cell renal cell carcinoma (ccRCC) using data from public platform and in vitro experiments. Our results revealed that the expression of JMJD4 is higher in cancerous tissue than in normal tissues $(p<0.001)$. High expression of JMJD4 is associated with a poor overall survival (OS) of ccRCC as compared with low expression of JMJD4 ( $p=0.015)$. JMJD4 showed significant relevance with M stage $(p=0.016)$, gender $(p=0.003)$, OS $(0.018)$, disease-specific survival (DSS) (0.007), and percussion free interval (PFI) (0.041). Univariate and multivariate Cox analyses demonstrated that high JMJD4 expression had independent predictive value for OS in ccRCC patients (hazard ratio $(\mathrm{HR})=1.563,95 \%$ confidence interval $(\mathrm{CI})$ $=1.055-2.316$, and $p=0.026)$. Besides, in vitro experiments confirmed that high expression of JMJD4 can significantly promote the invasion ability $(p<0.001)$, cloning ability $(p<0.001)$, and proliferation $(p<0.001)$ of renal cell carcinoma. In summary, high JMJD4 expression may be a prognostic marker in patients with kidney cancer.
\end{abstract}

\section{Introduction}

Renal cell carcinoma (RCC) is a common malignant tumor of the genitourinary system. It can be mainly divided into three subtypes, clear cell renal cell carcinoma (ccRCC), chromophobe, and papillary renal cell carcinoma, which comprise $65-70 \%, 15-20 \%$, and $5-7 \%$ of all RCCs [1, 2]. RCC accounts for nearly $3 \%$ of all adult malignant tumors, and its incidence and related mortality have been increasing globally in the past decades $[3,4]$. Usually, some patients have been prompted to have advanced RCC at the time of diagnosis due to the insidious onset of RCC. This tricky feature leads to poor prognosis and high mortality of patients with RCC. In advanced RCC, increased resistance to targeted therapies which may be mediated by gene disorders, angiogenesis, and decreased intake of tyrosine kinase inhibitors by cancer cells is common. In view of the hidden onset of RCC and the high resistance rate of targeted drug therapy for advanced RCC, it is urgent to explore effective biomolecular markers to contribute to the early diagnosis and prognostic information for RCC.
The modification of histones is one of the epigenetic mechanisms that regulate gene expression without changing the genotype $[5,6]$. Hitherto, histone modifications that have been widely studied are histone methylation and acetylation modifications, which are closely related to the occurrence of many cancers $[7,8]$. Histone demethylase is one of the most important modification enzymes involved in the regulation of chromatin function by catalyzing the removal of the methyl group on the $\mathrm{N}$-terminal lysine residue of histone [9]. Many histone demethylases have been proven to promote the genesis and development of cancer, such as KDM3A, KDM4A, KDM4D, and JMJD6. Study has confirmed that KDM3A, which is highly expressed in colorectal cancer, is conducive to metastasis of colorectal cancer [10]. KDM3A has also been found to be increased in pancreatic tumors and promote cancer genesis by regulating expression of DCLK1 [11]. KDM4A could function as an oncogene in lung cancer by mediating expression of $\mathrm{Myc}$ through the $\mathrm{Wnt} / \beta$-catenin signaling pathway [12]. In our previous study, we found that KDM4D inhibition can 
reduce the proliferation and development of kidney cancer. In addition, JMJD6 has been confirmed to be related to the progression of kidney cancer recently [13]. JMJD4 is a newly discovered histone demethylase which is homologous to JMJD6. However, the relationship between JMJD4 and tumor, especially kidney cancer, has not been reported yet.

This study is aimed at evaluating the prognostic value of JMJD4 in kidney cancer. We also conducted in vitro experiments to evaluate the effect of JMJD4 on ccRCC cells. Our results demonstrated that JMJD4 may be a cancerpromoting gene, and high expression of JMJD4 portends a poor prognosis in these patients.

\section{Materials and Methods}

2.1. Data Acquisition. The gene expression data and clinical data of ccRCC patients (Workflow Type: FPKM) were acquired from the online TCGA website (https://portal.gdc .cancer.gov/). Immunohistochemistry staining of ccRCC tissues was downloaded from The Human Protein Atlas (https://www.proteinatlas.org/). Patients with missing data, unknown staging status, and unknown survival status were excluded. Finally, a total of 539 patients with ccRCC with clinical characteristics information were obtained.

2.2. Gene Ontology (GO) and Kyoto Encyclopedia of Genes and Genomes Pathway (KEGG) Enrichment Analysis. Differentially expressed genes in the low and high JMJD4 data set were identified and categorized into specific signaling pathways by GO (https://www.geneontology.org) and the KEGG database (http://www.genome.ad.jp/kegg/). The significant GO and KEGG pathways were identified by Fisher's exact test. Adjusted $p$ value $<0.05$ and $q$ value $<0.2$ were considered as statistically significant.

2.3. Cell Culture. Human kidney cancer cell line (Caki-1), purchased from American Tissue Culture Collection (ATCC; Manassas, VA, USA), were cultured in McCoy's $5 \mathrm{~A}$ (HyClone, Logan, UT, USA) medium at $37^{\circ} \mathrm{C}, 5 \% \mathrm{CO}_{2}$ in a humidified environment. All media are supplemented with $10 \%$ fetal bovine serum (Hyclone). Then, cells were transfected with pcDNA-3.1(+)-JMJD4 plasmid and control vector plasmid.

2.4. Quantitative Real-Time Polymerase Chain Reaction ( $q R T-P C R)$ Analysis. Caki-1 cells were harvested, and total RNA was extracted. Then, cDNA was synthesized by EntiLink $^{\mathrm{TM}} 1$ st Strand cDNA Synthesis Kit (ELK Biotechnology, Wuhan, China) according to the manufacturer's instruction. Quantitative real-time PCR was performed using the StepOne ${ }^{\mathrm{TM}}$ Real-Time PCR (Life technologies, Wuhan, China) with the following primer sequences: JMJD4 sense primer, 5'-AGATGGTGTTTGTGCCCAGT-3'; JMJD4 antisense primer, $\quad 5^{\prime}$-CATGTTGGCCAGGTTGAAGC- $3^{\prime} ; \beta$-actin sense primer, $5^{\prime}$-CATCATCCCTGCCTCTACTGG-3' ; and $\beta$-actin antisense primer, $5^{\prime}$-GTGGGTGTCGCTGTTG AAGTC-3'.
TABLE 1: Clinical characteristics of ccRCC patients.

\begin{tabular}{|c|c|c|}
\hline Characteristic & Levels & Overall \\
\hline$n$ & & 539 \\
\hline \multirow{4}{*}{ T stage, $n(\%)$} & $\mathrm{T} 1$ & $278(51.6 \%)$ \\
\hline & $\mathrm{T} 2$ & $71(13.2 \%)$ \\
\hline & $\mathrm{T} 3$ & $179(33.2 \%)$ \\
\hline & $\mathrm{T} 4$ & $11(2 \%)$ \\
\hline \multirow{2}{*}{$\mathrm{N}$ stage, $n(\%)$} & No & $241(93.8 \%)$ \\
\hline & N1 & $16(6.2 \%)$ \\
\hline \multirow{2}{*}{ M stage, $n(\%)$} & M0 & $428(84.6 \%)$ \\
\hline & M1 & $78(15.4 \%)$ \\
\hline \multirow{4}{*}{ Pathologic stage, $n(\%)$} & Stage I & $272(50.7 \%)$ \\
\hline & Stage II & $59(11 \%)$ \\
\hline & Stage III & $123(22.9 \%)$ \\
\hline & Stage IV & $82(15.3 \%)$ \\
\hline \multirow{4}{*}{$\begin{array}{l}\text { Primary therapy outcome, } \\
n(\%)\end{array}$} & $\mathrm{PD}$ & $11(7.5 \%)$ \\
\hline & SD & $6(4.1 \%)$ \\
\hline & $\mathrm{PR}$ & $2(1.4 \%)$ \\
\hline & CR & $128(87.1 \%)$ \\
\hline \multirow{2}{*}{ Gender, $n(\%)$} & Female & $186(34.5 \%)$ \\
\hline & Male & $353(65.5 \%)$ \\
\hline \multirow{3}{*}{ Race, $n(\%)$} & Asian & $8(1.5 \%)$ \\
\hline & $\begin{array}{c}\text { Black or African } \\
\text { American }\end{array}$ & $57(10.7 \%)$ \\
\hline & White & $467(87.8 \%)$ \\
\hline \multirow{2}{*}{ Age, $n(\%)$} & $\leq 60$ & $269(49.9 \%)$ \\
\hline & $>60$ & $270(50.1 \%)$ \\
\hline \multirow{4}{*}{ Histologic grade, $n(\%)$} & G1 & $14(2.6 \%)$ \\
\hline & G2 & $235(44.3 \%)$ \\
\hline & G3 & $207(39 \%)$ \\
\hline & G4 & $75(14.1 \%)$ \\
\hline Age, median (IQR) & & $61(52,70)$ \\
\hline
\end{tabular}

2.5. Colony Formation Assay. Caki-1 cells were digested and resuspended in medium and seeded on a 6 -well plate at a density of 500 cells per well. The culture was maintained in McCoy's 5A medium and terminated when visible clones appeared. Next, the cells were harvested and fixed with $4 \%$ paraformaldehyde for 15 minutes. The cells were then stained with crystal violet and dried at room temperature. Finally, the number of clones which contains more than 50 cells was counted under the microscope.

2.6. Tumor Invasion Assays. Caki-1 cells were trypsinized, resuspended in serum-free culture medium, and counted. The upper chamber membranes of Transwell were evenly covered with Matrigel (BD science, New Jersey, USA) and incubated for $1 \mathrm{~h}$ before the cells were seeded. After 24 hours of conventional culture, cells in the upper chamber were gently wiped off by a cotton swab. Each chamber was fixed 


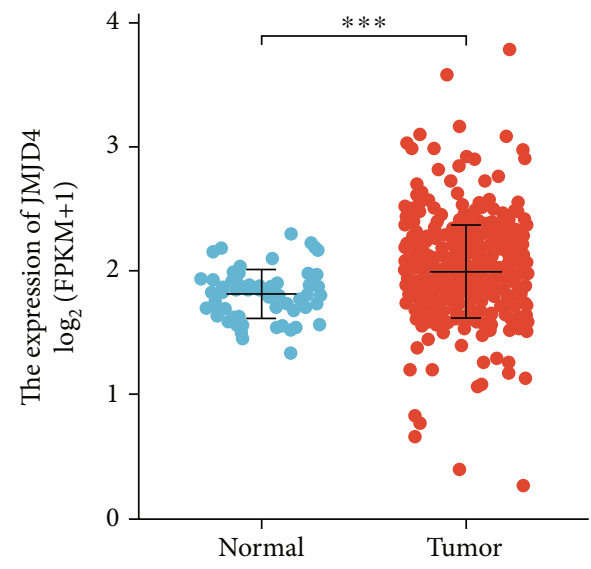

(a)

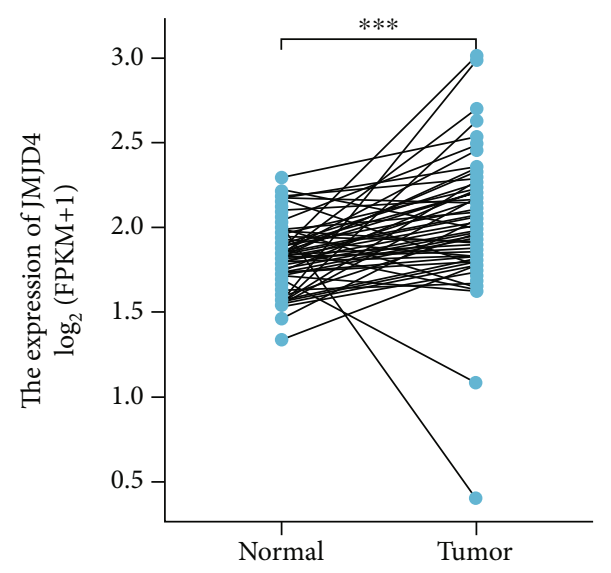

(b)
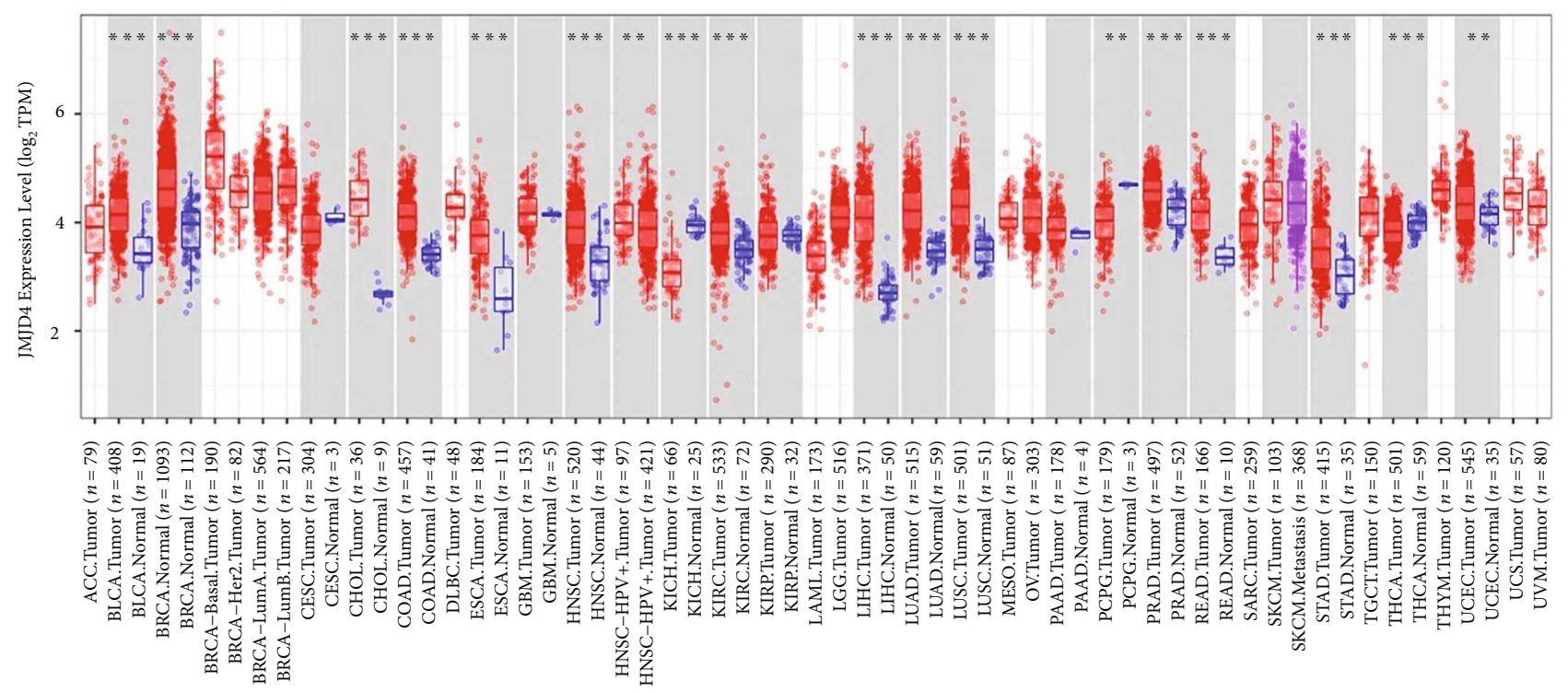

(c)

FIGURE 1: (a) JMJD4 expression in normal and tumor tissues. $* * *$ indicates a $p$ value $<0.001$. (b) JMJD4 expression in paired tissue. $* * *$ indicates a $p$ value $<0.001$. (c) JMJD4 expression in different types of human cancers in the TIMER database. $* *$ indicates a $p$ value $<0.01$, and $* * *$ indicates a $p$ value $<0.001$.

with methanol, stained with crystal violet, washed with PBS, and dried at room temperature. Six fields were randomly selected to take pictures under a microscope.

2.7. Wound-Healing Assays. Cells were harvested and seeded on six-well plates with $5 \times 10^{5}$ per well. To simulate a wound, the cell surface was scratched by a $10 \mu \mathrm{l}$ sterile plastic pipette tip with a straightedge when cells reached a confluent of $85 \%$ to $90 \%$. Then, the cells were cultured in serum-free medium and incubated at $37^{\circ} \mathrm{C}$. Overnight, wound images were collected under a microscope. The distance of the wound was measured and recorded, and the migration distance of cells in each group was calculated.

2.8. Cell Viability Assay. The proliferation of kidney cancer cells was determined by CCK-8 assays (Dojindo Molecular Technologies, Kumamoto, Japan). Generally, renal cancer cells were seeded into 96-well plates and incubated for $0 \mathrm{~h}$, $24 \mathrm{~h}, 48 \mathrm{~h}$, and $72 \mathrm{~h}$. Then, the medium in each well was discarded and replaced by $100 \mu \mathrm{l}$ of serum-free medium mixed with $10 \mu \mathrm{l}$ of CCK-8 under dark condition. After incubated for $2 \mathrm{~h}$, OD values at the absorbance of $470 \mathrm{~nm}$ were recorded.

2.9. Western Blot Analysis. Caki-1 cells were lysed by RIPA lysis buffer (Thermo Fisher Scientific Inc., MA, USA) mixed with protease and phosphatase inhibitors. Then, proteins were separated by SDS-PAGE and transferred to PVDF membranes. After transfer, membranes were put into 5\% bovine serum albumin and sealed at $25^{\circ} \mathrm{C}$ for 2 hours. Next, membranes were washed thrice with TBST on a shaker for 5 minutes each time and incubated with TBST diluted primary JMJD4 antibody (AP1030a, Abcepta, San Diego, CA, USA) and anti- $\beta$-actin antibody (ab8227, Abcam, Cambridge, 
TABLE 2: Correlation between the expression of JMJD4 and clinical characteristics.

\begin{tabular}{|c|c|c|c|}
\hline Characteristic & Low expression of JMJD4 & High expression of JMJD4 & $p$ \\
\hline$n$ & 269 & 270 & \\
\hline T stage, $n(\%)$ & & & 0.369 \\
\hline $\mathrm{T} 1$ & $147(27.3 \%)$ & $131(24.3 \%)$ & \\
\hline $\mathrm{T} 2$ & $37(6.9 \%)$ & $34(6.3 \%)$ & \\
\hline $\mathrm{T} 3$ & $80(14.8 \%)$ & $99(18.4 \%)$ & \\
\hline $\mathrm{T} 4$ & $5(0.9 \%)$ & $6(1.1 \%)$ & \\
\hline $\mathrm{N}$ stage, $n(\%)$ & & & 0.224 \\
\hline N0 & $136(52.9 \%)$ & $105(40.9 \%)$ & \\
\hline N1 & $6(2.3 \%)$ & $10(3.9 \%)$ & \\
\hline M stage, $n(\%)$ & & & 0.016 \\
\hline M0 & $231(45.7 \%)$ & $197(38.9 \%)$ & \\
\hline M1 & $30(5.9 \%)$ & $48(9.5 \%)$ & \\
\hline Pathologic stage, $n(\%)$ & & & 0.083 \\
\hline Stage I & $144(26.9 \%)$ & $128(23.9 \%)$ & \\
\hline Stage II & $34(6.3 \%)$ & $25(4.7 \%)$ & \\
\hline Stage III & $58(10.8 \%)$ & $65(12.1 \%)$ & \\
\hline Stage IV & $32(6 \%)$ & $50(9.3 \%)$ & \\
\hline Primary therapy outcome, $n(\%)$ & & & 0.762 \\
\hline $\mathrm{PD}$ & $4(2.7 \%)$ & $7(4.8 \%)$ & \\
\hline SD & $4(2.7 \%)$ & $2(1.4 \%)$ & \\
\hline PR & $1(0.7 \%)$ & $1(0.7 \%)$ & \\
\hline $\mathrm{CR}$ & $63(42.9 \%)$ & $65(44.2 \%)$ & \\
\hline Gender, $n(\%)$ & & & 0.003 \\
\hline Female & $76(14.1 \%)$ & $110(20.4 \%)$ & \\
\hline Male & $193(35.8 \%)$ & $160(29.7 \%)$ & \\
\hline Race, $n(\%)$ & & & 0.091 \\
\hline Asian & $5(0.9 \%)$ & $3(0.6 \%)$ & \\
\hline Black or African American & $21(3.9 \%)$ & $36(6.8 \%)$ & \\
\hline White & $240(45.1 \%)$ & $227(42.7 \%)$ & \\
\hline Age, $n(\%)$ & & & 0.111 \\
\hline$\leq 60$ & $144(26.7 \%)$ & $125(23.2 \%)$ & \\
\hline$>60$ & $125(23.2 \%)$ & $145(26.9 \%)$ & \\
\hline Histologic grade, $n(\%)$ & & & 0.073 \\
\hline G1 & $6(1.1 \%)$ & $8(1.5 \%)$ & \\
\hline G2 & $124(23.4 \%)$ & $111(20.9 \%)$ & \\
\hline G3 & $106(20 \%)$ & $101(19 \%)$ & \\
\hline G4 & $27(5.1 \%)$ & $48(9 \%)$ & \\
\hline OS event, $n(\%)$ & & & 0.018 \\
\hline Alive & $196(36.4 \%)$ & $170(31.5 \%)$ & \\
\hline Dead & $73(13.5 \%)$ & $100(18.6 \%)$ & \\
\hline DSS event, $n(\%)$ & & & 0.007 \\
\hline Alive & $223(42.2 \%)$ & $197(37.3 \%)$ & \\
\hline Dead & $41(7.8 \%)$ & $67(12.7 \%)$ & \\
\hline PFI event, $n(\%)$ & & & 0.041 \\
\hline Alive & $200(37.1 \%)$ & $178(33 \%)$ & \\
\hline Dead & $69(12.8 \%)$ & $92(17.1 \%)$ & \\
\hline Age, mean $\pm S D$ & $59.87 \pm 12.11$ & $61.39 \pm 12.05$ & 0.145 \\
\hline
\end{tabular}


TABLE 3: Logistic regression analysis of the association between JMJD4 expression and clinical characteristics.

\begin{tabular}{|c|c|c|c|}
\hline Characteristics & Total $(N)$ & Odds ratio(OR) & $p$ value \\
\hline T stage (T3 \& T4 vs. T1 \& T2) & 539 & $1.378(0.967-1.967)$ & 0.077 \\
\hline $\mathrm{N}$ stage (N1 vs. N0) & 257 & $2.159(0.776-6.522)$ & 0.148 \\
\hline M stage (M1 vs. M0) & 506 & $1.876(1.151-3.102)$ & 0.013 \\
\hline Gender (male vs. female) & 539 & $0.573(0.399-0.819)$ & 0.002 \\
\hline Age $(>60$ vs. $\leq 60)$ & 539 & $1.336(0.953-1.876)$ & 0.093 \\
\hline Histologic grade (G3 \& G4 vs. G1 \& G2) & 531 & $1.224(0.870-1.723)$ & 0.246 \\
\hline Race (Black or African American \& White vs. Asian) & 532 & $1.679(0.408-8.255)$ & 0.481 \\
\hline Primary therapy outcome (PR \& CR vs. PD \& SD) & 147 & $0.917(0.325-2.541)$ & 0.866 \\
\hline Pathologic stage (stage III \& stage IV vs. stage I \& stage II) & 536 & $1.487(1.048-2.113)$ & 0.027 \\
\hline
\end{tabular}

MA, USA) at $4^{\circ} \mathrm{C}$. Overnight, membranes were washed by TBST and incubated with horseradish peroxidase- (HRP-) labeled secondary antibody (Jackson $1: 2000$ ) for $2 \mathrm{~h}$ at $25^{\circ} \mathrm{C}$. Finally, membranes were reacted with electrochemical luminescence regents (Thermo Fisher Scientific Inc., MA, USA) and photographed in dark room.

2.10. Statistical Analysis. Clinical and RNA-seq data were organized and analyzed through R software 3.6.3 (Auckland, New Zealand) according to previous studies [14]. The box plots are used to analyze the expression level of JMJD4 between normal tissue and cancerous tissue among patients with various kinds of cancers including ccRCC. The relationship between JMJD4 expression and clinical characteristics of ccRCC patients was assessed by Wilcoxon signed rank test and Logistic regression. Univariate and multivariate Cox analyses were used to determine whether JMJD is an independent prognosis factor. Overall survival (OS), diseasespecific survival (DSS), and progression-free interval (PFI) curves were plotted by the Kaplan-Meier method and compared with the log rank test. The diagnostic value of JMJD4 was assessed by plotting the received operating characteristic (ROC) curve and calculating the area under the curve. Significances were computed by Graphpad Prism 7.0 (Graphpad Software, Inc., CA, USA).

\section{Results}

3.1. Clinical Characteristics of ccRCC Patients. Totally, clinical data of 539 patients with ccRCC were downloaded from the official site of TCGA and analyzed. The characteristics of 539 patients are shown in Table 1. There are 353 male participants (65.5\%) and 186 female participants (34.5\%) with a median age of patients is 61 . In terms of Fuhrman grade, 14 patients were grade I (2.6\%), 235 patients were grade II (44.3\%), 207 patients were grade III (39\%), and 75 patients were grade IV (14.1\%).

3.2. Expression of JMJD4 in ccRCC Tissues. JMJD4 was significantly higher in ccRCC tissues $(p<0.001)$ than in normal tissues both in paired and unpaired box plot analysis (Figures 1(a) and 1(b)). The expression of JMJD4 was also higher in bladder cancer, breast cancer, cholangiocarcinoma, colon cancer, renal cancer, lung cancer, etc., than in normal tissue $(p<0.01$ or $p<0.001$, Figure $1(\mathrm{c}))$.

3.3. Correlation of High Expression of JMJD4 with Clinical Characteristics. The relationships between JMJD4 and clinical characteristics of ccRCC patients are displayed in Table 2. JMJD4 showed significant relevance with $M$ stage $(p=0.016)$, gender $(p=0.003)$, OS (0.018), DSS (0.007), and PFI (0.041). As shown in Table 3, Logistic regression analysis confirmed that high expression of JMJD4 was significantly associated with $\mathrm{M}$ stage (M1 vs. $\mathrm{M} 0$ : $\mathrm{OR}=1.876$, $95 \%$ confidence interval $(\mathrm{CI})=1.151-3.102$, and $p=0.013$ ), pathological stage (stage III and stage IV vs. stage I and stage II, $\mathrm{OR}=1.48795 \%, \mathrm{CI}=1.048-2.113$, and $p=0.027$ ), and gender (male vs. female, $\mathrm{OR}=0.573,95 \% \mathrm{CI}=0.399-0.819$, and $p=0.002)$.

3.4. High Expression of JMJD Predicts a Worse Clinical Outcome. Online immunohistochemical staining images of JMJD4 in ccRCC patients are shown in Figure 2(a). We performed Kaplan-Meier survival analysis to assess the prognostic value of JMJD4, and results demonstrated that high JMJD4 expression was associated with poor OS $(p=0.015)$, DSS (0.005), and PFI (0.02) as shown in Figures 2(b)-2(d). Subgroup analysis by different clinical characteristics demonstrated that high JMJD4 expression was significantly associated with poor OS in patients with advance Fuhrman grade $(p=0.009)$, female patients $(p=0.026)$, patients from T1 to T3 stage $(p=0.016)$, patients no greater than 60 years old $(p=0.002)$, and patients with low or normal hemoglobin $(p=0.021)$ as shown in Figures 3(a)-3(e). High level of JMJD4 is also connected with poor PFI in patients from T1 to T3 stage $(p=0.021)$ and poor DSS in patients no greater than 60 years old $(p=0.008)$ and female patients $(p=0.02)$.

Univariate Cox regression analysis manifested that high JMJD4 expression was significantly correlated with poor OS $(\mathrm{HR}=1.466,95 \% \mathrm{CI}=1.084-1.983$, and $p=0.013)$. Multivariate cox regression further validated that JMJD4 has independent prognostic value in patients with ccRCC (Table 4 and Figure $4, \mathrm{HR}=1.563,95 \% \mathrm{CI}=1.055-2.316$, and $p=0.026)$.

3.5. Diagnostic Value of JMJD4 in ccRCC Patients. The area under the ROC was 0.717 , which indicates a high diagnostic 


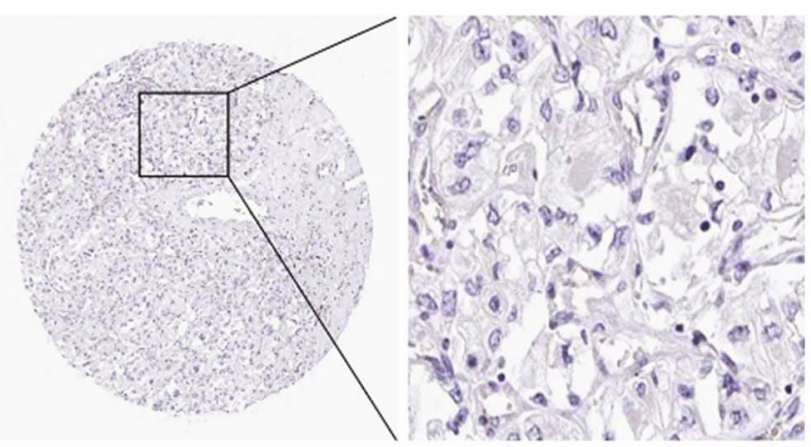

(A)

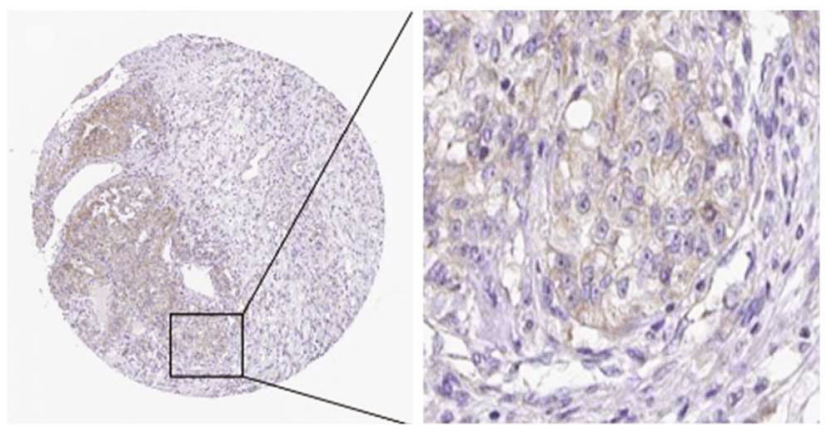

(C)

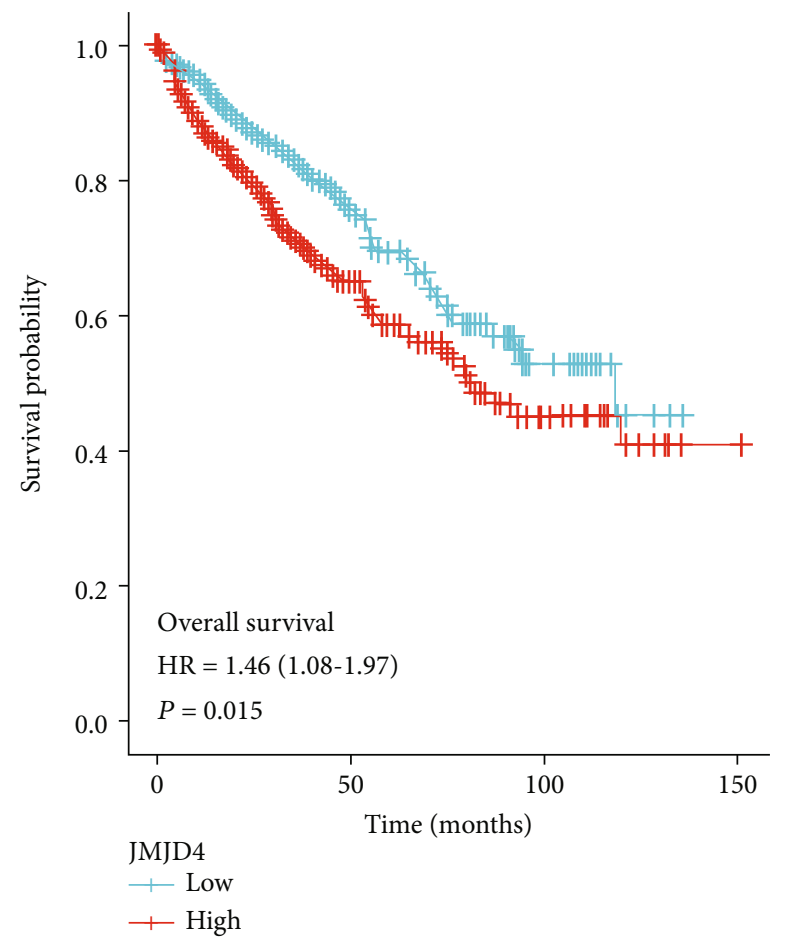

(b)

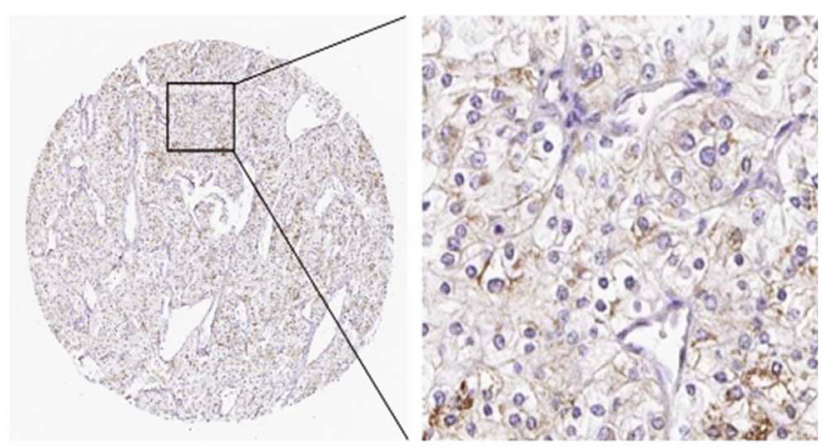

(B)

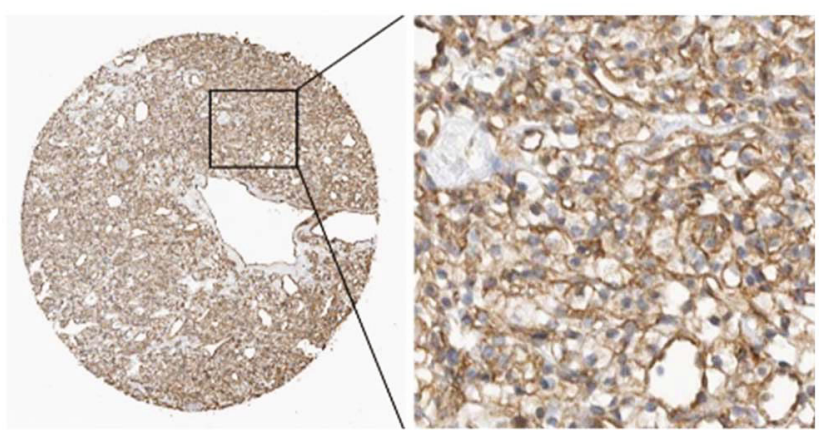

(D)

(a)

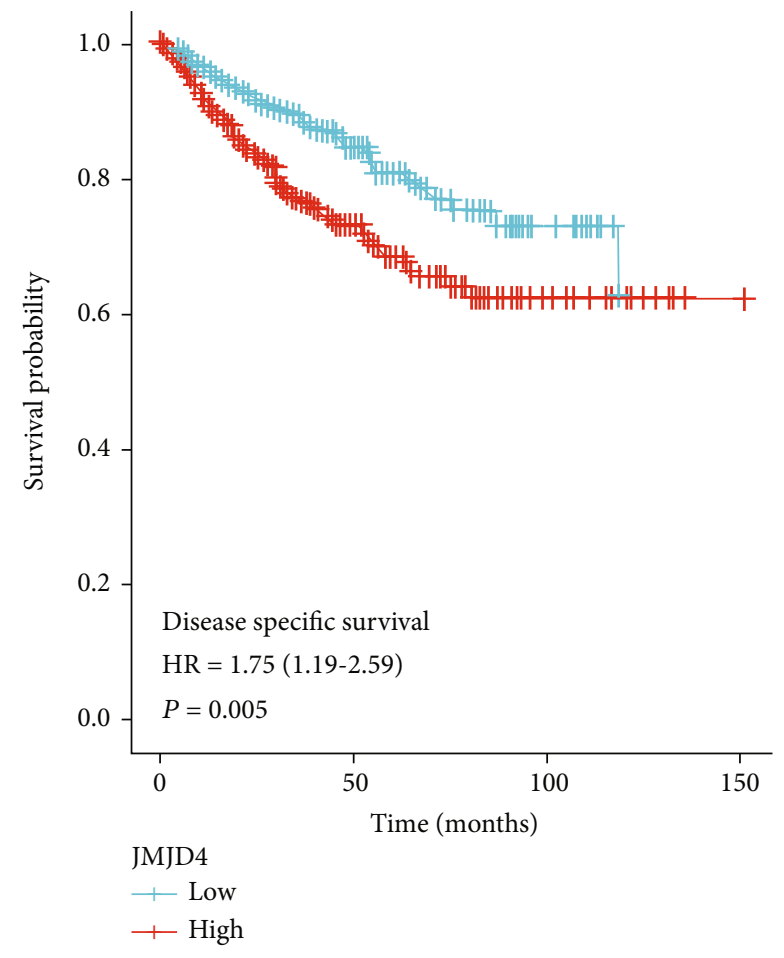

(c)

Figure 2: Continued. 


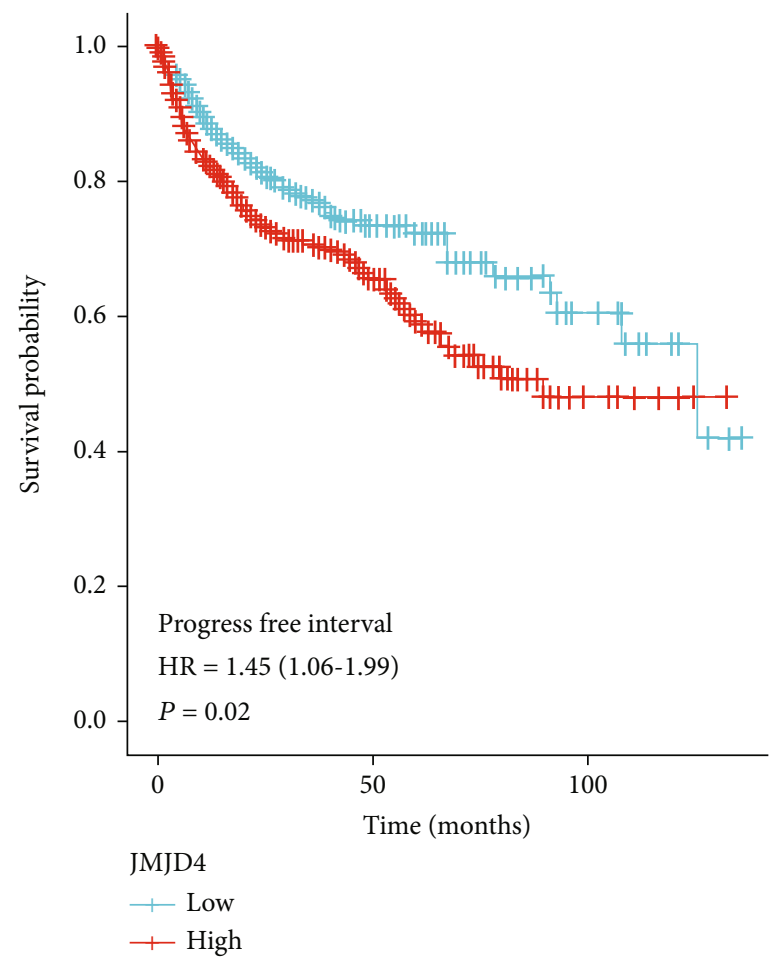

(d)

FIGURE 2: High expression level of JMJD4 portends a worse prognosis and outcome. (a) Online representative images of IHC staining of JMJD4 in cancerous tissue microarrays. A, minimum expression; B, low expression; C, moderate expression; and D, high expression. Scale bar $=100 \mu \mathrm{m}$. (b-d) Kaplan-Meier curves of OS, DSS, and PFI.

value of JMJD for ccRCC patients (Figure 5(a)). The 1-, 3-, and 5-year survival probability of ccRCC patients are shown in Figure 5(b) by constructing a nomogram with the combination of JMJD4 and other clinical characteristics.

3.6. Genes Enriched in Different Signaling Pathways. We analyzed the RNA-seq data from TCGA database. Volcano map of differentially expressed genes is shown in Figure 6(a), and more genes were downregulated. The result of KEGG pathway enrichment is shown in Figure 6(b). KEGG analysis has shown that three most enriched pathways were estrogen signaling pathway, Staphylococcus aureus infection, and neuroactive ligand-receptor pathway. There are 31 genes enriched in tumor-related pathways as shown in Figure 6(c), suggesting that JMJD4 is closely related to cancer development. GO analysis and KEGG pathway enrichment were performed to investigate genes correlated with JMJD4 associated with ccRCC. Pathways that meet $p$ value $<0.05$ and $q$ value $<0.2$ are considered to be statistically significant. In biological process (BP) of GO analysis, genes were mainly associated with cornification, keratinization, keratinocyte differentiation, epidermal cell differentiation, and skin development (Figure 7(a)). Significant pathways in cellular component (CC) included keratin filament, extracellular region, intermediate filament, intermediate filament cytoskeleton, and extracellular region part as shown in Figure 7(b). Most enriched genes in molecular function (MF) include transmembrane transporter activity, channel activity, passive transmembrane transporter activity, transporter activity, and ion transmembrane transporter activity (Figure $7(\mathrm{c})$ ). Genes enriched in pathway mentioned above include DSG3, KRT4, KRT5, KRT6, KRT14, KRT15, KRT16, and IVL.

3.7. JMJD4 Promoted Renal Cancer Cell Proliferation, Invasion, and Colony Formation. To exploit the role of JMJD4 in renal cell proliferation and development, plasmid containing pcDNA-3.1(+)-JMJD4 was transfected into the Caki-1 cell line. The Transwell invasion assay and colony formation assay were then used to unravel the effect of JMJD4 on cell invasion and colony formation, and the results showed that JMJD4 significantly facilitated the invasion and colony formation ability of Caki- 1 cells $(p<0.001$ , Figure $8(\mathrm{a}))$. The efficiency of overexpression was verified by qRT-PCR and western blot (Figures $8(\mathrm{~b})$ and $8(\mathrm{c})$ ). As a result, western blot analysis and qRT-PCR showed that the expression of JMJD4 was significantly upregulated after transfection of pcDNA-3.1(+)-JMJD4. To investigate the effect that JMJD4 exerts on renal cell proliferation, we performed CCK-8 assays. The results showed that overexpression of JMJD4 in Caki-1 cells promoted the renal cell growth $(p<0.001$, Figure $8(\mathrm{~d}))$.

\section{Discussion}

JumonjiC (JmjC) domain-containing histone demethylase (JMJD) is a protein family that plays an important role in 
Histologic grade: G3\&G4

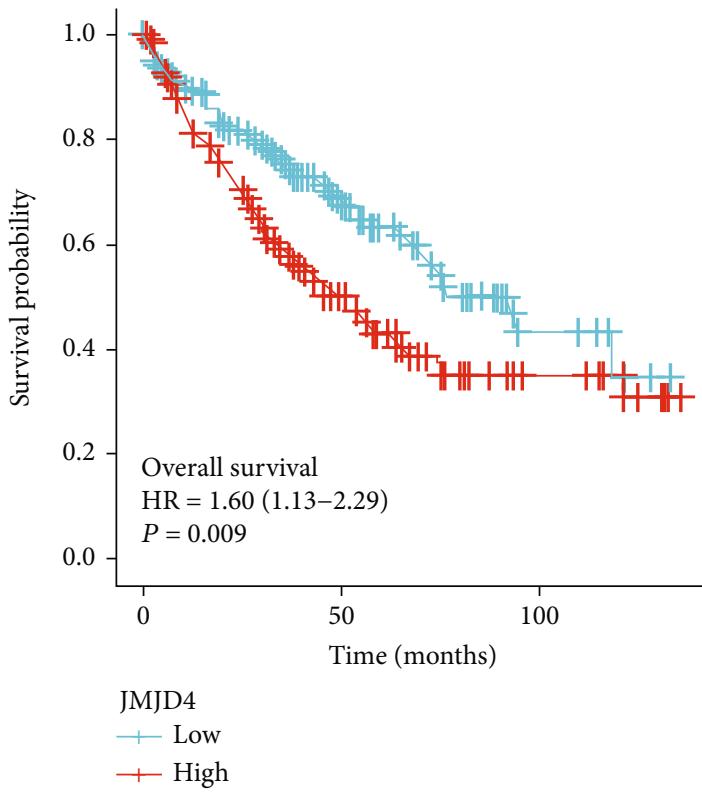

(a)

T stage: T1\&T2\&T3

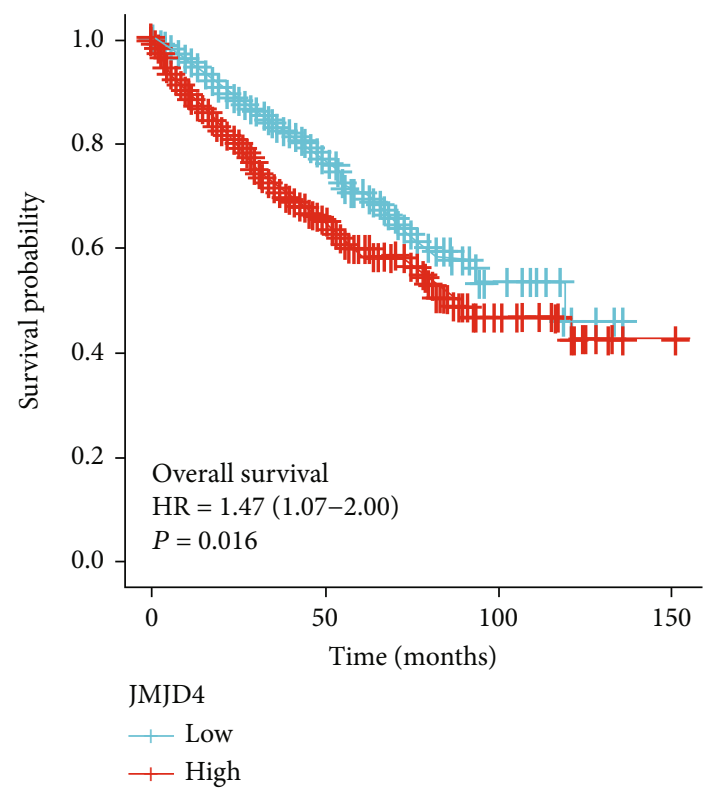

(c)
Gender: Female

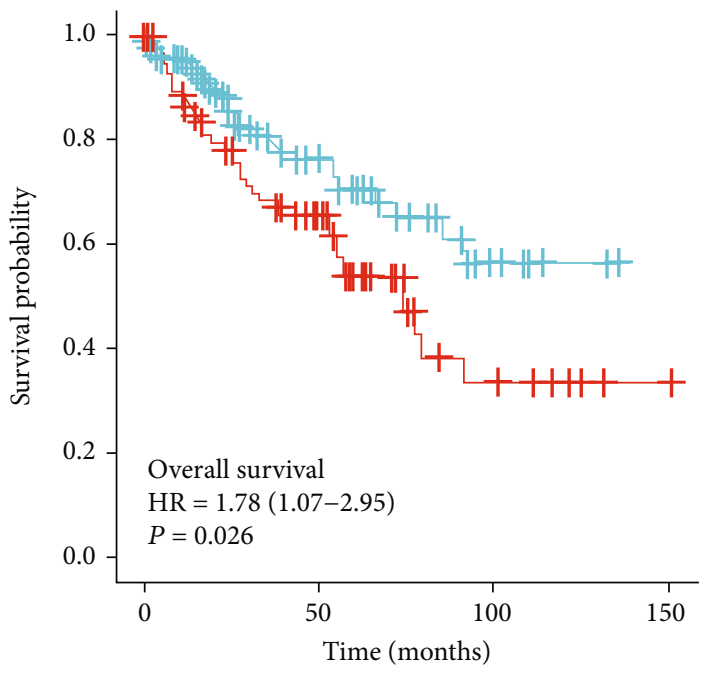

JMJD4

+ Low

(b)

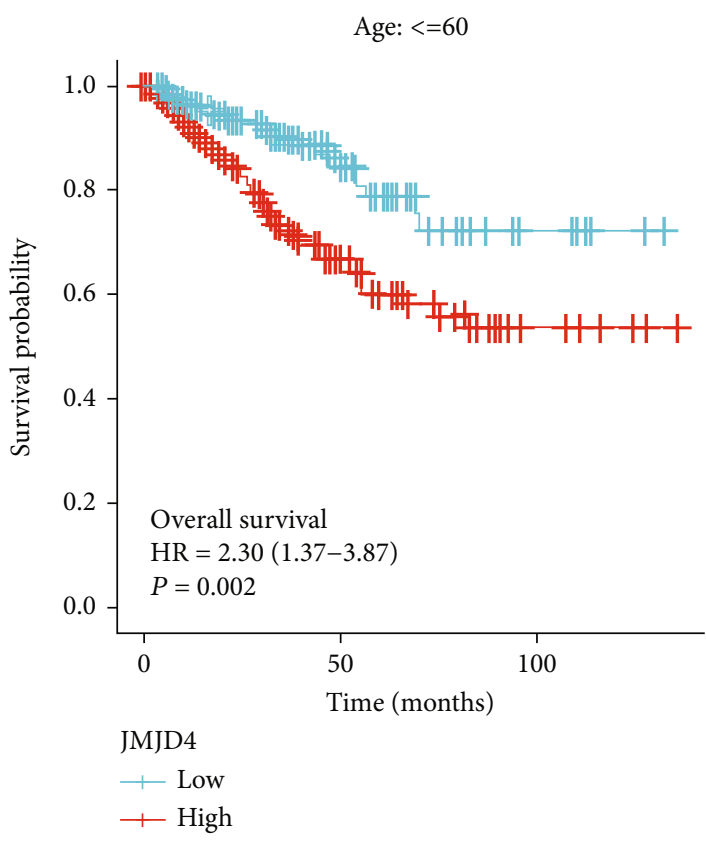

(d)

FIgUre 3: Continued. 


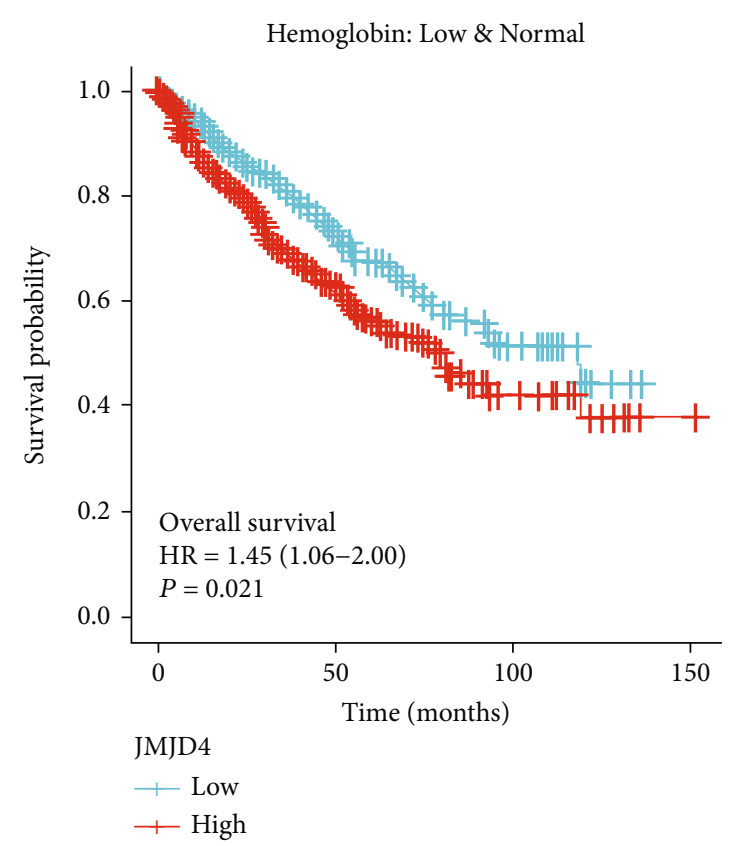

(e)

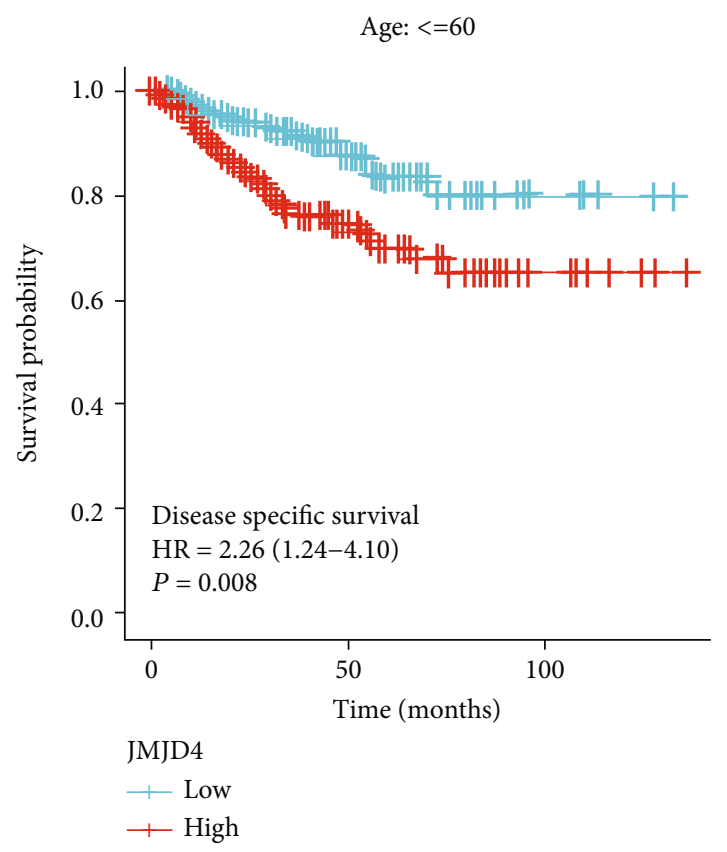

(g)

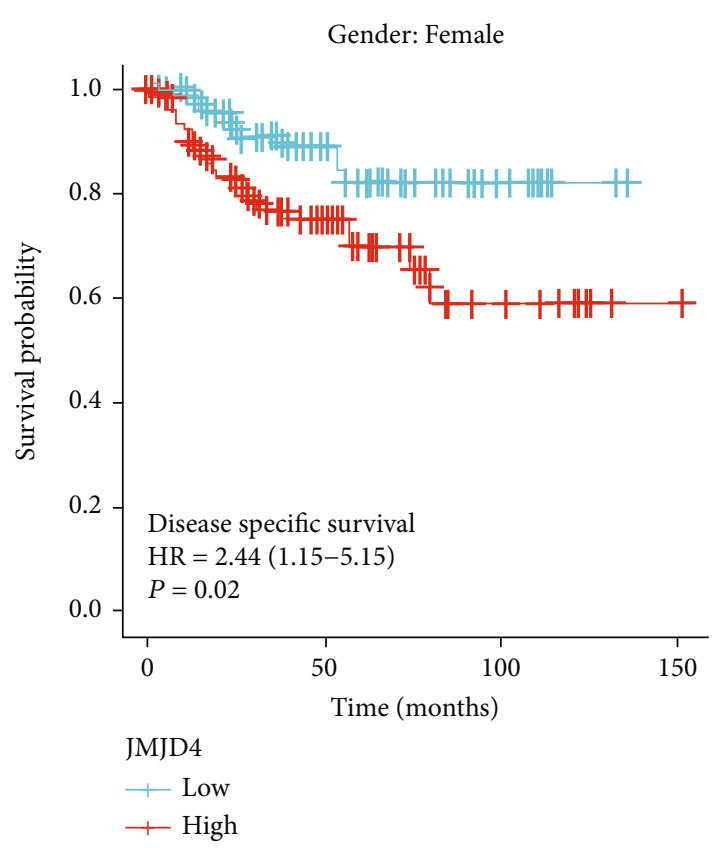

(f)

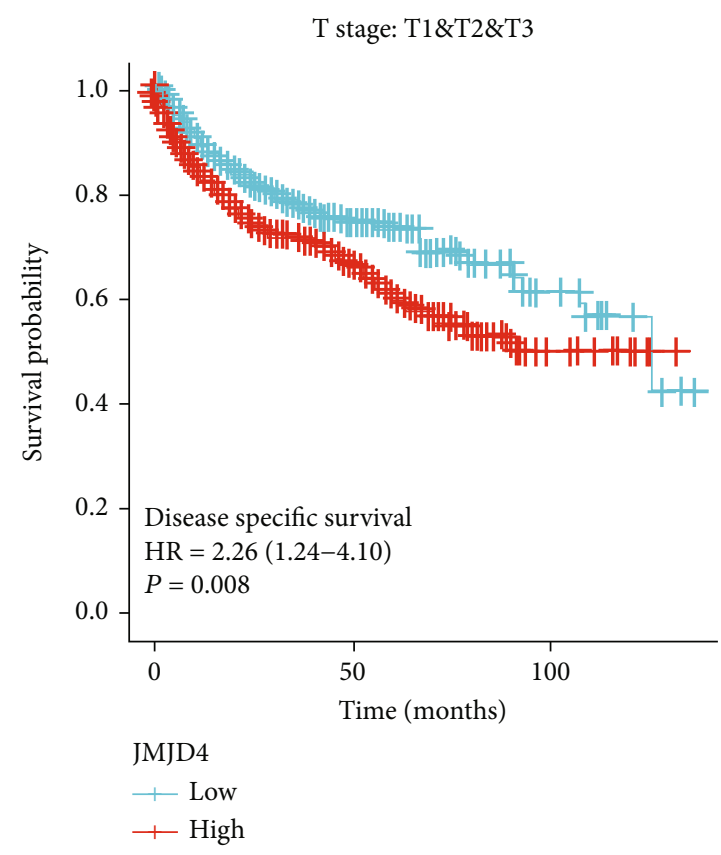

(h)

FIGURE 3: (a-e) Subgroup analysis of overall survival for patients in advanced Fuhrman grade, female patients, patients in T1 to T3 stage, patients age no greater than 60, and patients with low or normal hemoglobin. (f, g) Subgroup analysis of DSS for patients with ccRCC in female patients and patients age no greater than 60. (h) Subgroup analysis of PFI for patients with ccRCC in T1 to T3.

cancer growth and development by regulating gene transcription and chromatin structure. Among the members of family, JMJD6 was reported to be tumorigenic factor which involves in various kinds of cancers such as prostate cancer [15], breast cancer [16, 17], melanoma [18], colon cancer [19], and lung cancer [20]. JMJD4 is a paralog of JMJD6 which shares 34\% sequence with JMJD6 [21]. Knockout of the JMJD6 gene in mice will significantly reduce the proliferation of NIH3T fibroblasts, while knocking out JMJD4 will cause the same effect by RNA interference [22]. Study has confirmed that JMJD4-knockout embryos are born at a normal Mendelian ratio which proved that JMJD4 is unessential in embryogenesis [23]. Currently, only one study has reported that JMJD4 expression could be a prognostic indicator for patients with colon cancer [24]. The interaction between JMJD4 and other tumors still needs more research to reveal. Therefore, we investigate the relationship between JMJD4 and renal cancer. 
TABLE 4: Cox regression analyses of clinical characteristics associated with overall survival.

\begin{tabular}{|c|c|c|c|c|c|}
\hline \multirow{2}{*}{ Characteristics } & \multirow{2}{*}{ Total $(N)$} & \multicolumn{2}{|c|}{ Univariate analysis } & \multicolumn{2}{|c|}{ Multivariate analysis } \\
\hline & & Hazard ratio $(95 \% \mathrm{CI})$ & $p$ value & Hazard ratio $(95 \% \mathrm{CI})$ & $p$ value \\
\hline T stage & 539 & & & & \\
\hline $\mathrm{T} 1 \& \mathrm{~T} 2$ & 349 & Reference & & & \\
\hline $\mathrm{T} 3 \& \mathrm{~T} 4$ & 190 & $3.228(2.382-4.374)$ & $<0.001$ & $1.869(1.171-2.983)$ & 0.009 \\
\hline Histologic grade & 531 & & & & \\
\hline G1 \& G2 & 249 & Reference & & & \\
\hline G3 \& G4 & 282 & $2.702(1.918-3.807)$ & $<0.001$ & $1.704(1.037-2.801)$ & 0.036 \\
\hline JMJD4 & 539 & & & & \\
\hline Low & 270 & Reference & & & \\
\hline High & 269 & $1.466(1.084-1.983)$ & 0.013 & $1.563(1.055-2.316)$ & 0.026 \\
\hline $\mathrm{N}$ stage & 257 & & & & \\
\hline No & 241 & Reference & & & \\
\hline $\mathrm{N} 1$ & 16 & $3.453(1.832-6.508)$ & $<0.001$ & $1.678(0.819-3.438)$ & 0.157 \\
\hline $\mathrm{M}$ stage & 506 & & & & \\
\hline M0 & 428 & Reference & & & \\
\hline M1 & 78 & $4.389(3.212-5.999)$ & $<0.001$ & $2.950(1.832-4.750)$ & $<0.001$ \\
\hline Age & 539 & & & & \\
\hline$\leq 60$ & 269 & Reference & & & \\
\hline$>60$ & 270 & $1.765(1.298-2.398)$ & $<0.001$ & $1.684(1.095-2.590)$ & 0.018 \\
\hline Gender & 539 & & & & \\
\hline Female & 186 & Reference & & & \\
\hline Male & 353 & $0.930(0.682-1.268)$ & 0.648 & & \\
\hline
\end{tabular}

\begin{tabular}{ccccc}
\hline Characteristics & $\mathrm{N}(\%)$ & $\mathrm{HR}(95 \% \mathrm{CI})$ & $P$ value \\
\hline T stage & 539 & $1.869(1.171-2.983)$ & 0.009 \\
Histologic grade & 531 & $1.704(1.037-2.801)$ & 0.036 \\
JMJD4 & 539 & $1.563(1.055-2.316)$ & 0.026 \\
N stage & 257 & $1.678(0.819-3.438)$ & 0.157 \\
M stage & 506 & $2.950(1.832-4.750)$ & & 0.001 \\
Age & 539 & $1.684(1.095-2.590)$ & 1 & 2 \\
\hline
\end{tabular}

FIgURE 4: Forest plot of the multivariate Cox regression analysis in kidney cancer.

In this study, we first use bioinformatics analysis to assess the role of JMJD4 in ccRCC from online public database such as TCGA and TIMER. The results of box plot analysis demonstrate that high expression of JMJD4 is a common feature of multiple human cancers, including ccRCC. And Logistic regression analysis indicated that high JMJD4 is closely related to M stage, gender, and prognosis in ccRCC patients. Then, to evaluate the prognostic value of
JMJD4 in ccRCC patients, we performed Kaplan-Meier survival analysis, and results showed that patients with high JMJD4 expression had poor OS, DSS, and PFI. Moreover, Cox regression analysis confirmed that high JMJD4 expression has independent prognostic value for OS in ccRCC patients. Other clinicopathological characteristics are also related to the poor OS of ccRCC, including advanced Fuhrman grade, $\mathrm{M}$ stage, $\mathrm{T}$ stage, and age, as shown in Figure 4. 


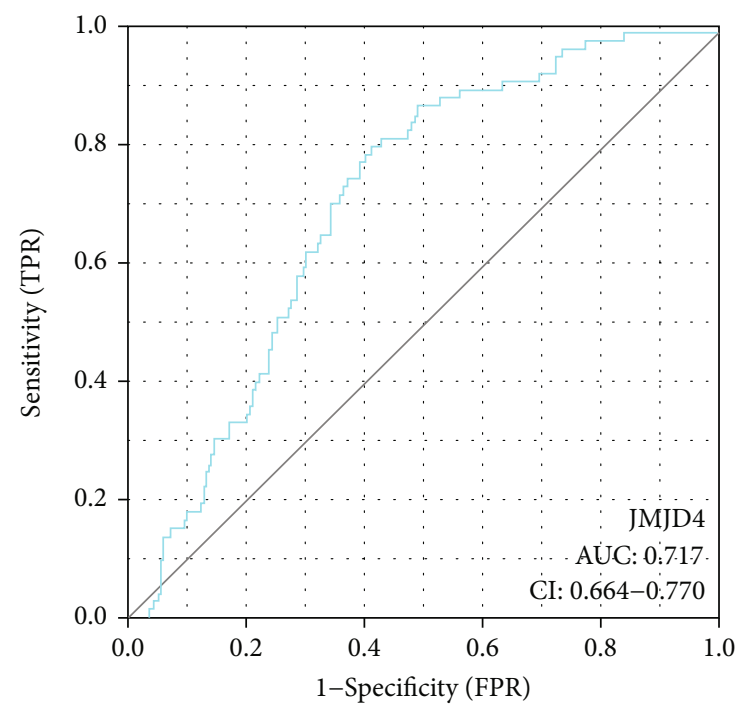

(a)

Points
T stage
N stage
M stage
Gender
Age
Histologic grade
Total Points
Linear Predictor
1-year Survival Probability
3-year Survival Probability
5-year Survival Probability

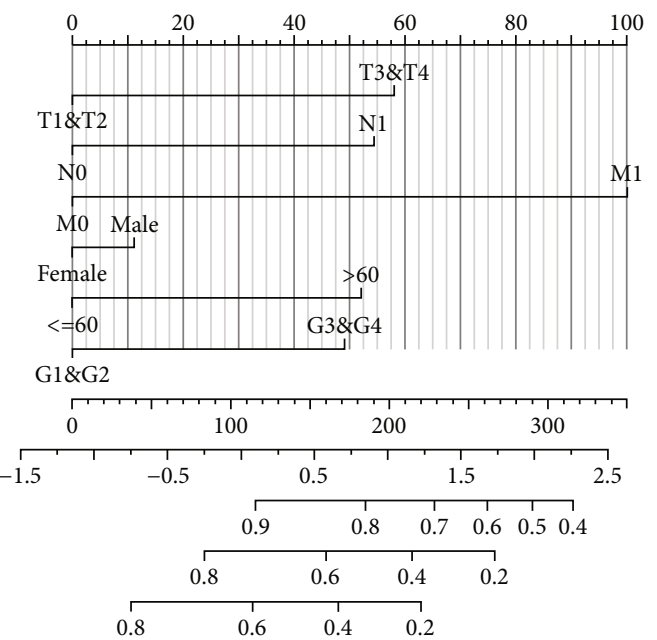

(b)

FIgURE 5: (a) ROC curve for JMJD4 in ccRCC patients. (b) Nomogram for predicting probability of patients with 1-, 3-, and 5-year overall survival.

Next, we performed ROC analysis and compute the AUC. The results suggest that high JMJD4 expression has certain diagnostic value in ccRCC patients (Figure 5(a)). Besides, to help practitioners predict the risk of individual death and optimize treatment options, we also constructed a prognostic nomogram (Figure 5(b)).

To further evaluate the mechanism of high expression of JMJD4 in promoting the progression of renal cancer, we performed GO and KEGG analysis. We found that the high JMJD4 expression was associated with collecting duct acid secretion, neuroactive ligand-receptor interaction, and fat digestion and absorption pathway in KEGG analysis. GO analysis shows that keratinization, keratinocyte differentiation, and epidermal cell differentiation were most enriched genes in BP. Skin development extracellular region, intermediate filament, and intermediate filament cytoskeleton were most enriched pathways in CC, while transmembrane trans- porter activity, channel activity, and passive transmembrane transporter activity were most enriched pathways in MF. The main genes enriched in these pathways include DSG3, KRT5, KRT6, and KRT14. DSG3 was reported to promote the growth and invasion of cancer cells [25]. KRT5 deficiency can prevent the migration of ovarian cancer cells, which prompts that it may act as an oncogene [26]. KRT6 is associated with notch1 signaling and contributes the progression of renal cancer [27]. KRT14 was proved to be a key regulator in metastasis of breast cancer [28]. The above data shows that the genes related to JMJD4 are mainly cancer-promoting genes, indicating that JMJD4 may promote the invasion and progression of cancer. Furthermore, to clarify the effect of JMJD4 on kidney cancer, we transfected the pcDNA-3.1(+)-JMJD4 plasmid into Caki-1 cells and verified the overexpression by qRT-PCR and western blot (Figures 8(a) and 8(b)). Then, we performed Transwell 


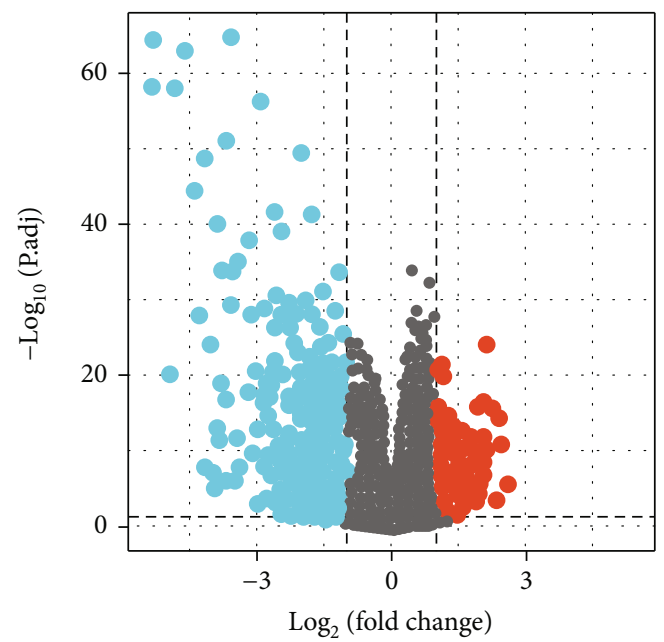

(a)

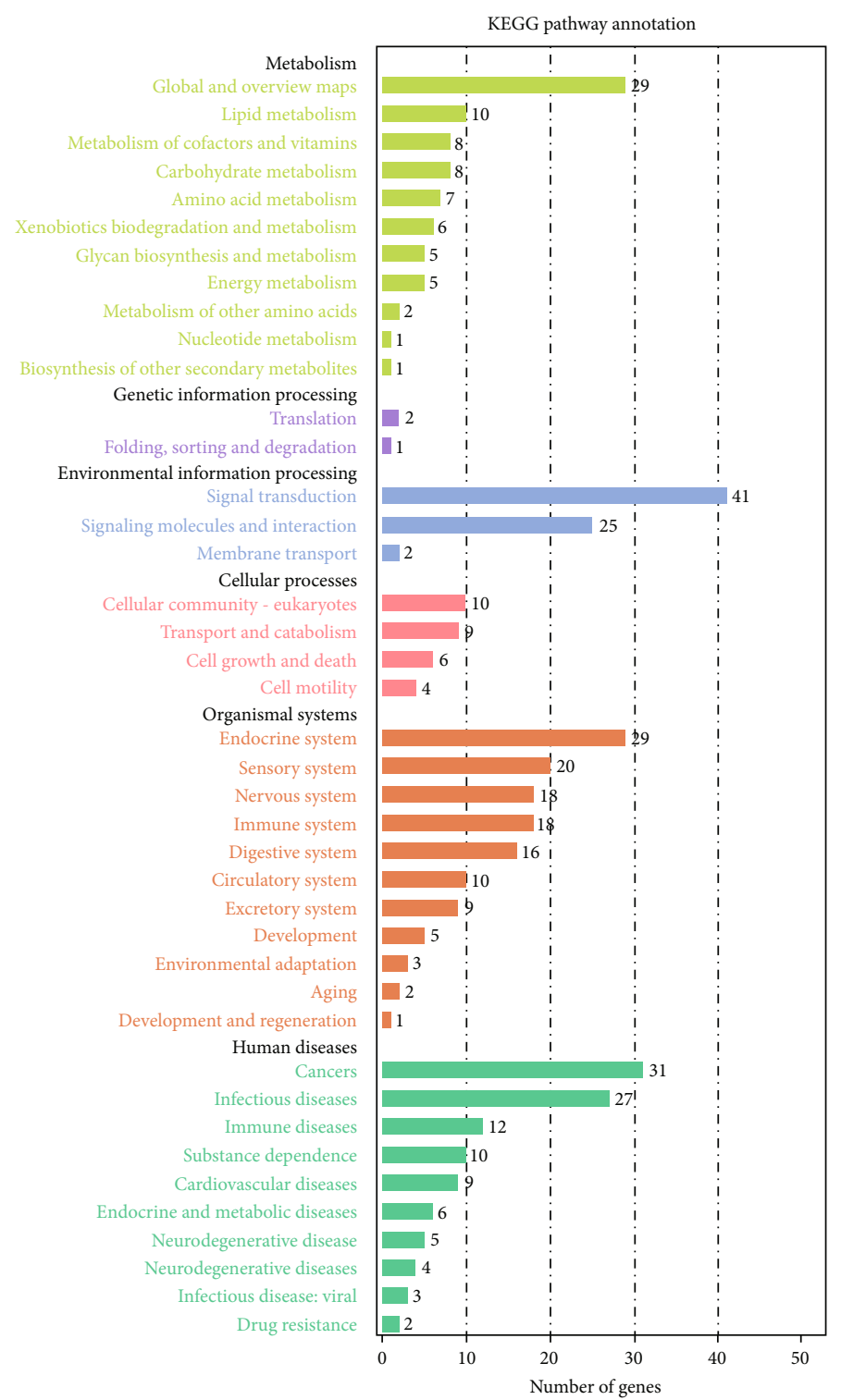

(b)

Figure 6: Continued. 


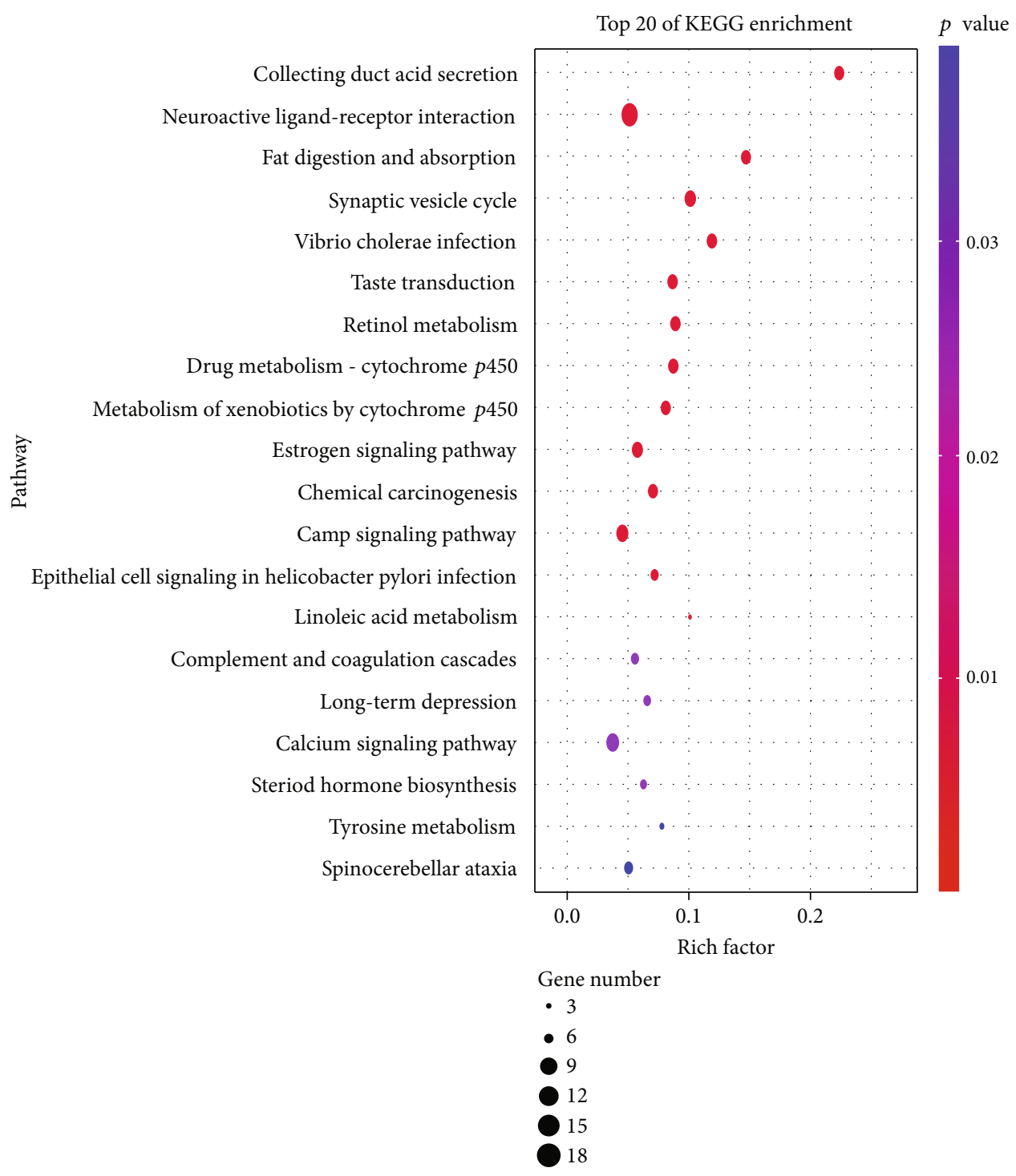

(c)

FIGURE 6: (a) Volcano map of the differentially expressed genes. (b) KEGG pathway analysis showed that genes mainly enriched in signal transduction and cancer disease. (c) Bubble diagram of top 20 most enriched genes in KEGG analysis. 


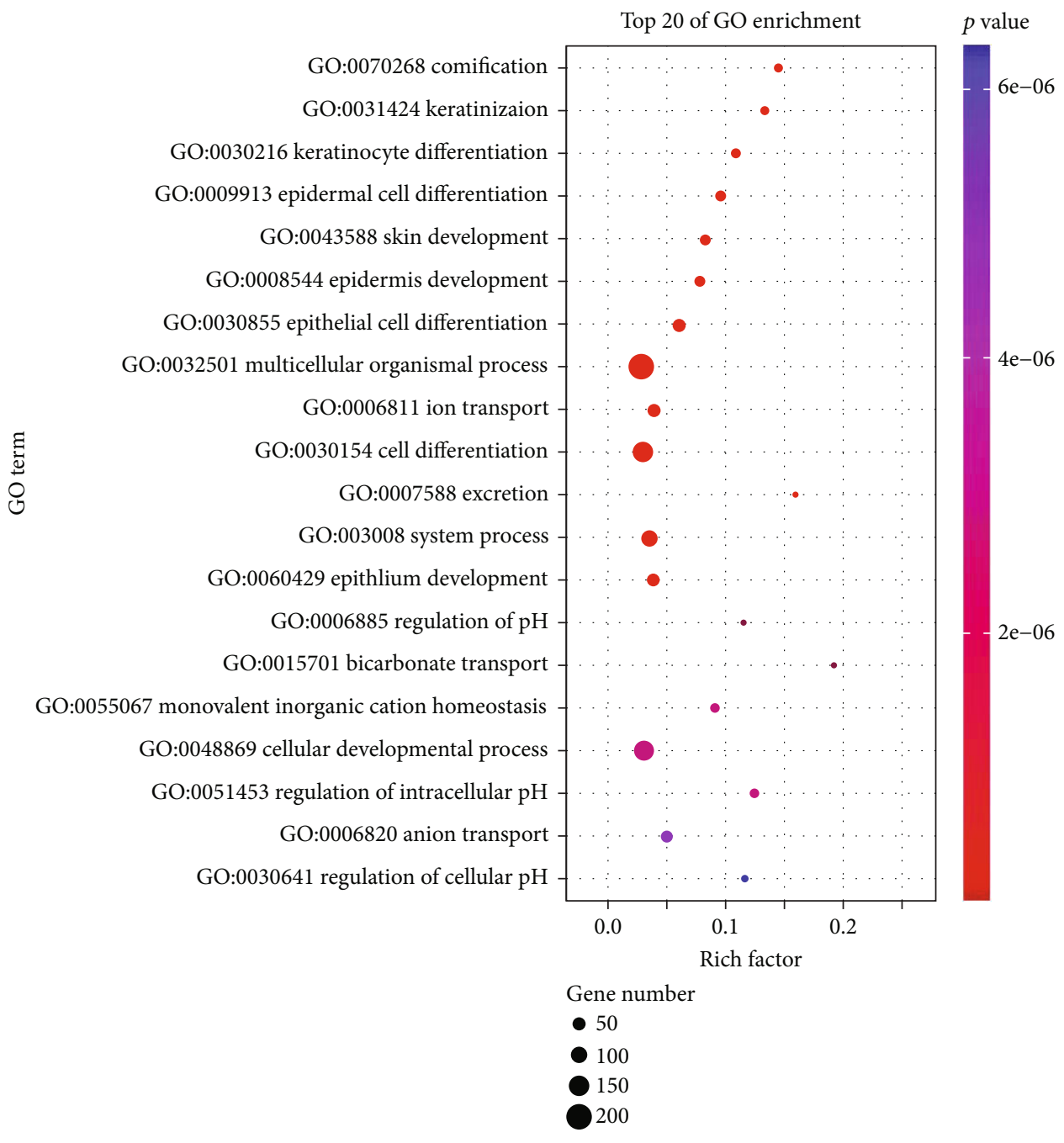

(a)

FIgURE 7: Continued. 


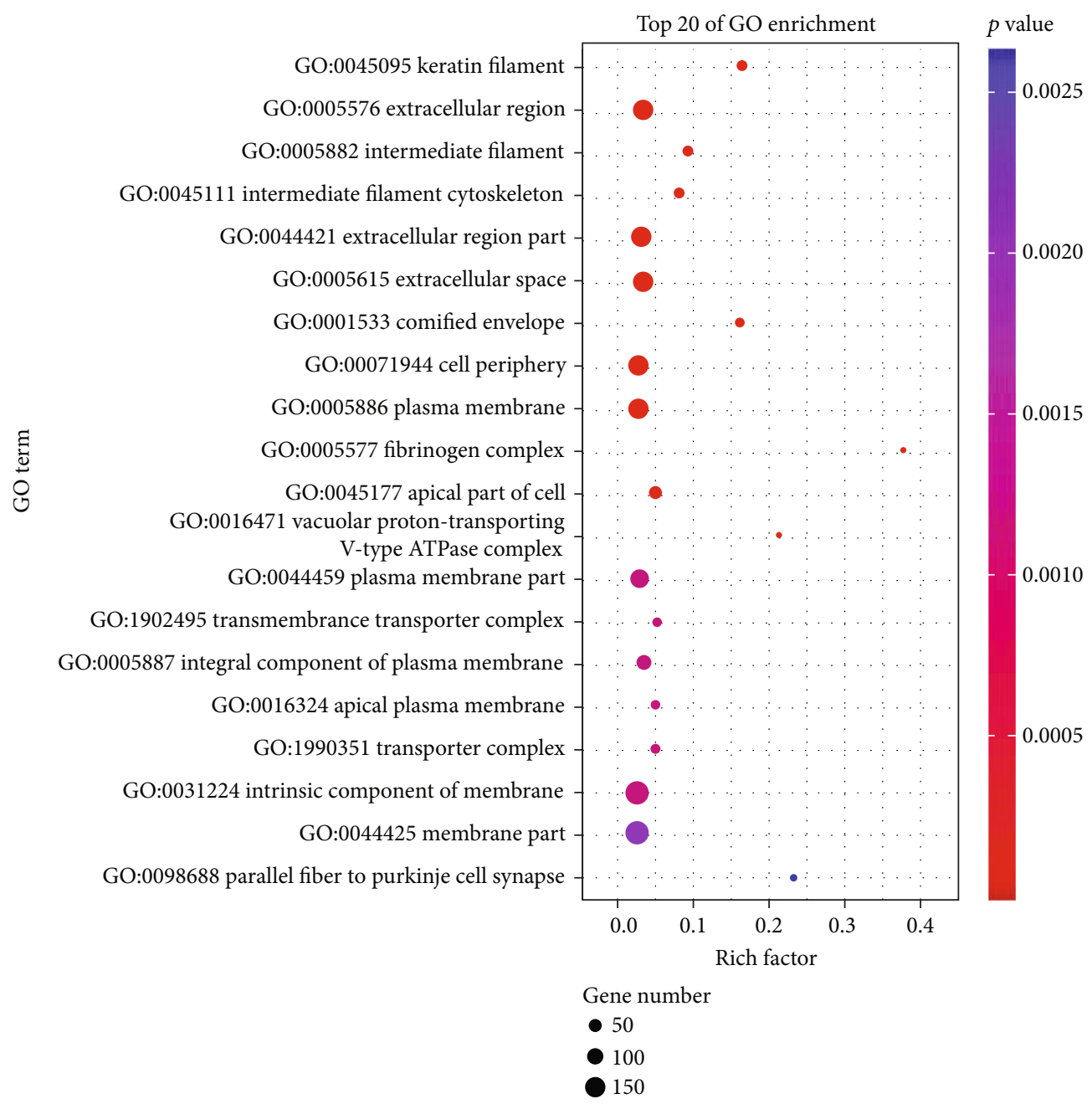

(b)

Figure 7: Continued. 


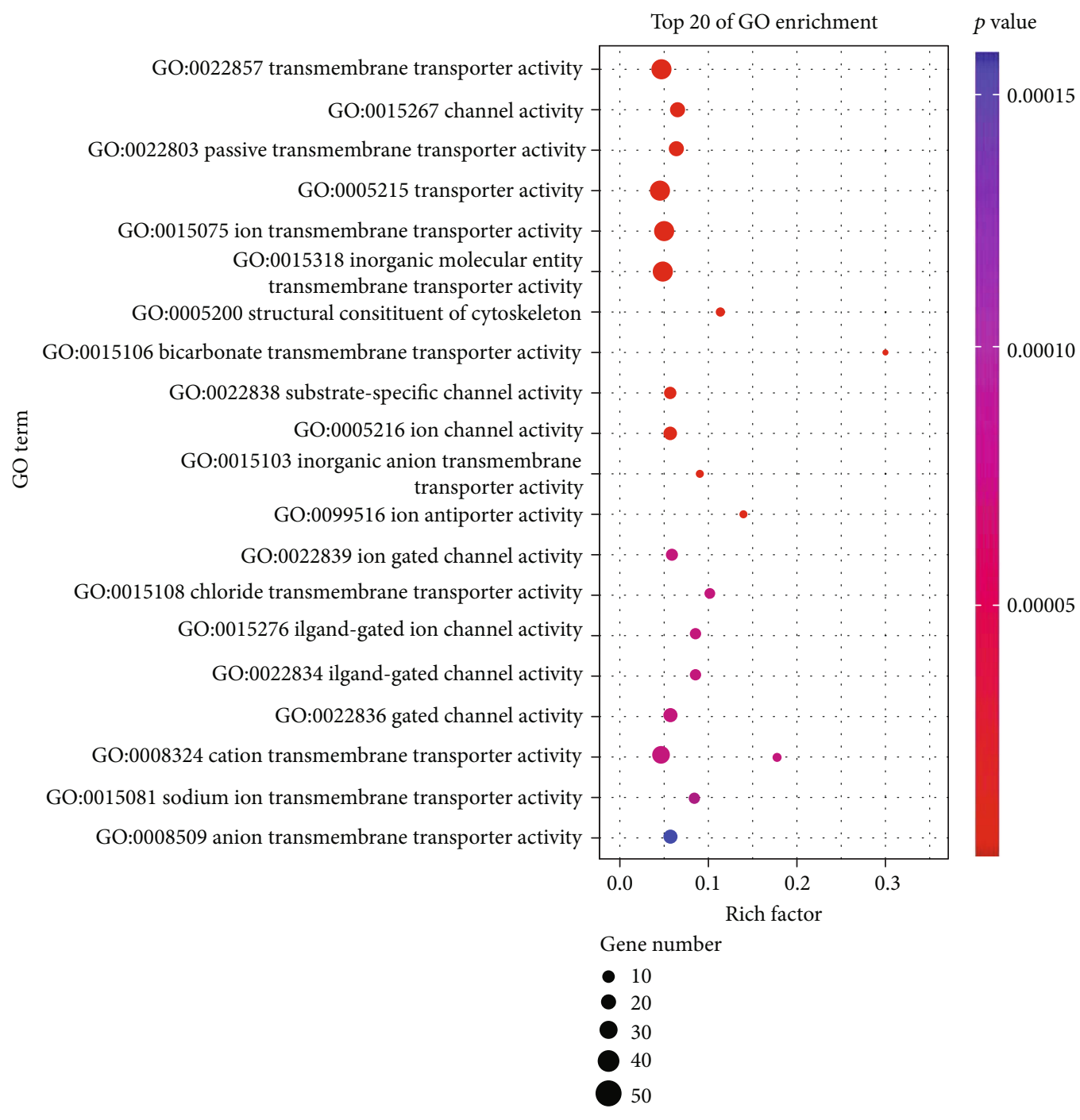

(c)

Figure 7: GO analysis. (a-c) Bubble diagram of top 20 most enriched genes in BP, CC, and MF analysis. 


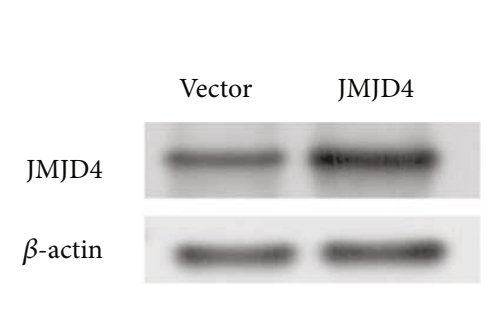

(a)

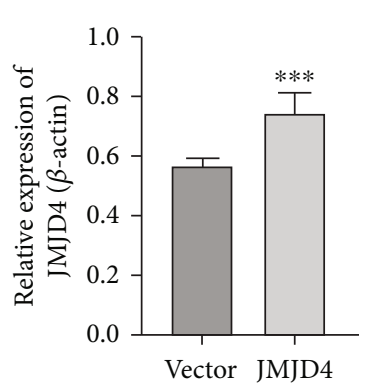

JMJD4

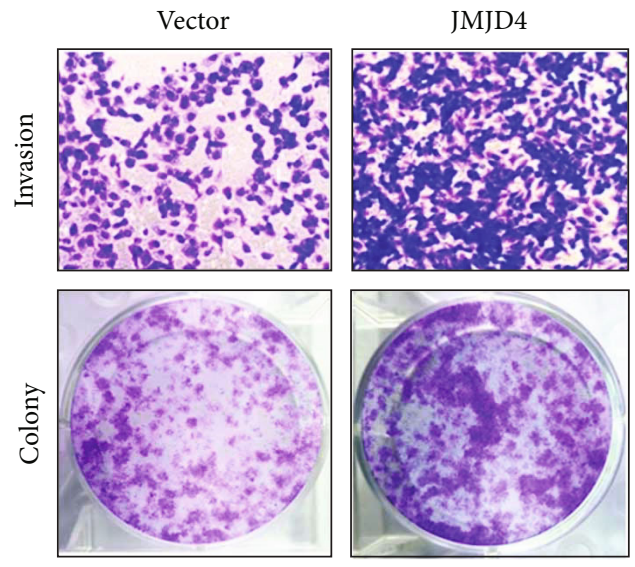

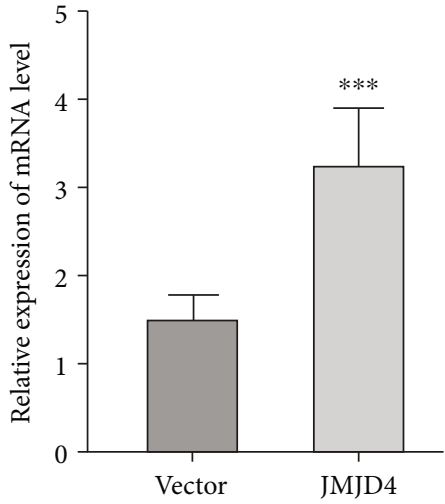

(b)
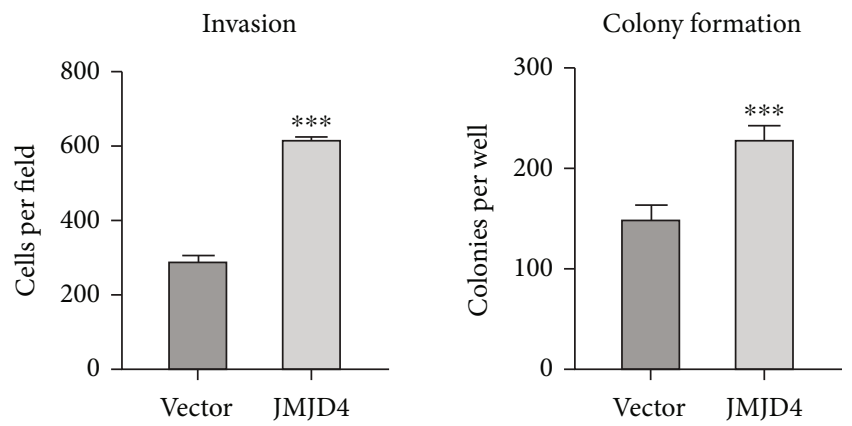

(c)

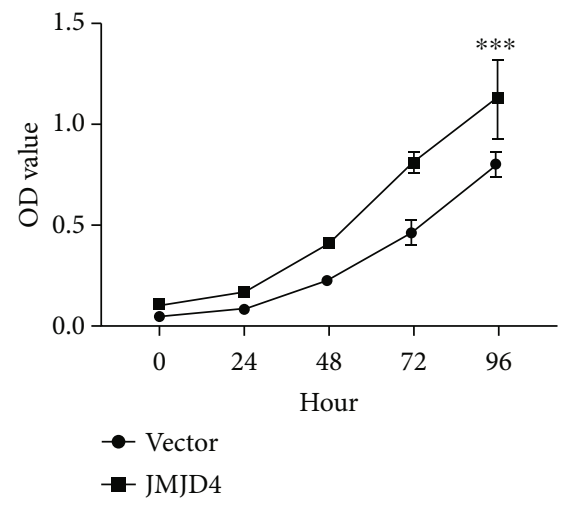

(d)

FIGURE 8: JMJD4 promoted cell proliferation, colony formation, and invasion in kidney cancer cells. (a, b) JMJD4 expression was validated by western blot and qRT-PCR assay. $* * *$ indicates a $p$ value $<0.001$. (c) Overexpressed JMJD4 promotes the invasion and colony formation capability of Caki-1 cells. $* * *$ indicates a $p$ value $<0.001$. (d) CCK- 8 proliferation curve of Caki- 1 cells. $* * *$ indicates a $p$ value $<0.001$.

invasion experiments and plate colony formation experiments. The results showed that JMJD4 overexpression significantly promoted the invasiveness and colony formation ability of Caki- 1 cells (Figures $8(c), p<0.001$ ). We also conducted the CCK-8 experiment, and the results demonstrate that JMJD4 can significantly promote the proliferation of Caki-1 cells (Figures 8(d), $p<0.001$ ). The above in vitro data prove that JMJD4 acts as a cancer-promoting gene in renal cancer cells. One limitation of our research is that we did not conduct in vivo experiments for verifica- tion. Subsequent experiments can be performed on animal experiments to verify the cancer-promoting effect of JMJD4 in vivo.

In summary, our study is the first research that focuses on the role of JMJD4 played in ccRCC. We validated the predictive value and diagnostic value of JMJD4 in ccRCC patients, as well as the effect of JMJD4 on promoting the invasion and proliferation of ccRCC cells. Our results showed that high expression of JMJD can be a potential prognostic marker for ccRCC patients. 


\section{Data Availability}

The datasets generated during the current study are available from the corresponding author on reasonable request.

\section{Conflicts of Interest}

The authors declare no conflict of interest.

\section{Authors' Contributions}

Hao Yan and Yewei Bao contributed equally to this work.

\section{Acknowledgments}

This research was granted by the Shanghai Science and Technology Development Foundation (17JC1400904).

\section{Supplementary Materials}

Supplementary 1. Supplemental material 1: details of the enriched genes in KEGG analysis.

Supplementary 2. Supplemental material 2: details of the enriched genes in biological process of GO analysis.

Supplementary 3. Supplemental material 3: details of the enriched genes in molecular function of GO analysis.

Supplementary 4. Supplemental material 4: details of the enriched genes in cellular component of GO analysis.

\section{References}

[1] K. Inamura, "Renal cell tumors: understanding their molecular pathological epidemiology and the 2016 WHO classification," International Journal of Molecular Sciences, vol. 18, no. 10, p. 2195, 2017.

[2] J. R. Srigley, B. Delahunt, J. N. Eble et al., "The International Society of Urological Pathology (ISUP) Vancouver classification of renal neoplasia," The American Journal of Surgical Pathology, vol. 37, no. 10, pp. 1469-1489, 2013.

[3] D. Labochka, B. Moszczuk, W. Kukwa, C. Szczylik, and A. M. Czarnecka, "Mechanisms through which diabetes mellitus influences renal cell carcinoma development and treatment: a review of the literature," International Journal of Molecular Medicine, vol. 38, no. 6, pp. 1887-1894, 2016.

[4] U. Capitanio, K. Bensalah, A. Bex et al., "Epidemiology of Renal Cell Carcinoma," European Urology, vol. 75, no. 1, pp. 74-84, 2019.

[5] R. Alam, H. M. Abdolmaleky, and J. R. Zhou, "Microbiome, inflammation, epigenetic alterations, and mental diseases," American Journal of Medical Genetics. Part B, Neuropsychiatric Genetics, vol. 174, no. 6, pp. 651-660, 2017.

[6] R. Lardenoije, A. Iatrou, G. Kenis et al., "The epigenetics of aging and neurodegeneration," Progress in Neurobiology, vol. 131, pp. 21-64, 2015.

[7] E. M. Michalak, M. L. Burr, A. J. Bannister, and M. A. Dawson, "The roles of DNA, RNA and histone methylation in ageing and cancer," Nature Reviews. Molecular Cell Biology, vol. 20, no. 10, pp. 573-589, 2019.
[8] J. E. Audia and R. M. Campbell, "Histone modifications and cancer," Cold Spring Harbor Perspectives in Biology, vol. 8, no. 4, article a019521, 2016.

[9] J. Zhang, L. Jing, M. Li, L. He, and Z. Guo, "Regulation of histone arginine methylation/demethylation by methylase and demethylase (review)," Molecular Medicine Reports, vol. 19, no. 5, pp. 3963-3971, 2019.

[10] J. Liu, T. Liang, and W. Zhangsun, "KDM3A is associated with tumor metastasis and modulates colorectal cancer cell migration and invasion," International Journal of Biological Macromolecules, vol. 126, pp. 318-325, 2019.

[11] P. Dandawate, C. Ghosh, K. Palaniyandi et al., "The histone demethylase KDM3A, increased in human pancreatic tumors, regulates expression of DCLK1 and promotes tumorigenesis in mice," Gastroenterology, vol. 157, no. 6, pp. 1646-1659.e11, 2019.

[12] S. Sun, F. Yang, Y. Zhu, and S. Zhang, "KDM4A promotes the growth of non-small cell lung cancer by mediating the expression of Myc via DLX5 through the Wnt/ $\beta$-catenin signaling pathway," Life Sciences, vol. 262, article 118508, 2020.

[13] C. Zhang, X. Lu, J. Huang et al., "Epigenome screening highlights that JMJD6 confers an epigenetic vulnerability and mediates sunitinib sensitivity in renal cell carcinoma," Clinical and Translational Medicine, vol. 11, no. 2, article e328, 2021.

[14] C.-Y. Jin, L. Du, A. H. Nuerlan, X.-L. Wang, Y.-W. Yang, and R. Guo, "High expression of RRM2 as an independent predictive factor of poor prognosis in patients with lung adenocarcinoma," Aging, vol. 13, no. 3, pp. 3518-3535, 2020.

[15] D. Tong, "The role of JMJD6/U2AF65/AR-V7 axis in castration-resistant prostate cancer progression," Cancer Cell International, vol. 21, no. 1, pp. 45-45, 2021.

[16] Y. F. Lee, L. D. Miller, X. B. Chan et al., "JMJD6 is a driver of cellular proliferation and motility and a marker of poor prognosis in breast cancer," Breast Cancer Research, vol. 14, no. 3, p. R85, 2012.

[17] A. Biswas, G. Mukherjee, P. Kondaiah, and K. V. Desai, "Both EZH2 and JMJD6 regulate cell cycle genes in breast cancer," BMC Cancer, vol. 20, no. 1, p. 1159, 2020.

[18] X. Liu, W. Si, X. Liu et al., "JMJD6 promotes melanoma carcinogenesis through regulation of the alternative splicing of PAK1, a key MAPK signaling component," Molecular Cancer, vol. 16, no. 1, pp. 175-175, 2017.

[19] F. Wang, L. He, P. Huangyang et al., "JMJD6 promotes colon carcinogenesis through negative regulation of $\mathrm{p} 53$ by hydroxylation," PLoS Biology, vol. 12, no. 3, pp. e1001819e1001819, 2014.

[20] J. Wan, W. Xu, J. Zhan et al., "PCAF-mediated acetylation of transcriptional factor HOXB9 suppresses lung adenocarcinoma progression by targeting oncogenic protein JMJD6," Nucleic Acids Research, vol. 44, no. 22, pp. 10662-10675, 2016.

[21] T. Feng, A. Yamamoto, S. E. Wilkins et al., "Optimal translational termination requires C4 Lysyl hydroxylation of eRF1," Molecular Cell, vol. 53, no. 4, pp. 645-654, 2014.

[22] Y. J. Hu and A. N. Imbalzano, "Global gene expression profiling of JMJD6- and JMJD4-depleted mouse NIH3T3 fibroblasts," Scientific Data, vol. 3, no. 1, 2016.

[23] H. Yoo, D. Son, Y. J. Lee, and K. Hong, "Mouse JMJD4 is dispensable for embryogenesis," Molecular Reproduction and Development, vol. 83, no. 7, pp. 588-593, 2016.

[24] Y. J. Ho, C. P. Shih, K. T. Yeh et al., "Correlation between high expression levels of jumonji domain-containing 4 and short 
survival in cases of colon adenocarcinoma," Biochemical and Biophysical Research Communications, vol. 503, no. 3, pp. 1442-1449, 2018.

[25] Y.-J. Chen, L.-Y. Lee, Y.-K. Chao et al., "DSG3 facilitates cancer cell growth and invasion through the DSG3-plakoglobinTCF/LEF-Myc/cyclin D1/MMP signaling pathway," PLoS One, vol. 8, no. 5, pp. e64088-e64088, 2013.

[26] Z. Zhang, K. Tu, F. Liu et al., "FoxM1 promotes the migration of ovarian cancer cell through KRT5 and KRT7," Gene, vol. 757, article 144947, 2020.

[27] J. Hu, L. C. Zhang, X. Song, J. R. Lu, and Z. Jin, "KRT6 interacting with notch1 contributes to progression of renal cell carcinoma, and aliskiren inhibits renal carcinoma cell lines proliferation in vitro," International Journal of Clinical and Experimental Pathology, vol. 8, no. 8, pp. 9182-9188, 2015.

[28] K. J. Cheung, V. Padmanaban, V. Silvestri et al., "Polyclonal breast cancer metastases arise from collective dissemination of keratin 14-expressing tumor cell clusters," Proceedings of the National Academy of Sciences of the United States of America, vol. 113, no. 7, pp. E854-E863, 2016. 